\title{
Surgery of highly eloquent gliomas primarily assessed as non-resectable: risks and benefits in a cohort study
}

\author{
Sandro M Krieg ${ }^{1 *}$, Lea Schnurbus ${ }^{1}$, Ehab Shiban ${ }^{1}$, Doris Droese $^{2}$, Thomas Obermueller ${ }^{1}$, Niels Buchmann ${ }^{1}$, \\ Jens Gempt ${ }^{1}$, Bernhard Meyer ${ }^{1}$ and Florian Ringel ${ }^{1}$
}

\begin{abstract}
Background: Today, the treatment of choice for high- and low-grade gliomas requires primarily surgical resection to achieve the best survival and quality of life. Nevertheless, many gliomas within highly eloquent cortical regions, e.g., insula, rolandic, and left perisylvian cortex, still do not undergo surgery because of the impending risk of surgery-related deficits at some centers. However, pre and intraoperative brain mapping, intraoperative neuromonitoring $(\mathrm{IOM})$, and awake surgery increase safety, which allows resection of most of these tumors with a considerably low rate of postoperatively new deficits.
\end{abstract}

Methods: Between 2006 and 2012, we resected 47 out of 51 supratentorial gliomas (92\%), which were primarily evaluated to be non-resectable during previous presentation at another neurosurgical department. Out of these, 25 were glioblastomas WHO grade IV (53\%), 14 were anaplastic astrocytomas WHO grade III (30\%), 7 were diffuse astrocytomas WHO grade II (15\%), and one was a pilocytic astrocytoma WHO grade I (2\%). All data, including pre and intraoperative brain mapping and monitoring (IOM) by motor evoked potentials (MEPs) were reviewed and related to the postoperative outcome.

Results: Awake surgery was performed in 8 cases (17\%). IOM was required in 38 cases (81\%) and was stable in 18 cases (47\%), whereas MEPs changed the surgical strategy in 10 cases (26\%). Thereby, gross total resection was achieved in 35 cases (74\%). Postoperatively, 17 of 47 patients (36\%) had a new motor or language deficit, which remained permanent in $8.5 \%$ (4 patients). Progression-free follow-up was 11.3 months (range: 2 weeks 64.5 months) and median survival was 14.8 months (range: 4 weeks - 20.5 months). Median Karnofsky Performance Scale was 85 before and 80 after surgery).

Conclusions: In specialized centers, most highly eloquent gliomas are eligible for surgical resection with an acceptable rate of surgery-related deficits; therefore, they should be referred to specialized centers.

Keywords: Language, Eloquent tumor, Rolandic region, Glioma, Neuromonitoring.

\section{Background}

For the treatment of high- and low-grade gliomas, surgery is an important part of a multimodal therapy [1-4]. Surgical tumor reduction has been shown to have a impact on survival and quality of life and, thus, has to be as extensive as possible [1,3-5]. Nonetheless, many gliomas within highly eloquent regions, especially within

\footnotetext{
* Correspondence: Sandro.Krieg@|rz.tum.de

${ }^{1}$ Department of Neurosurgery, Klinikum rechts der Isar, Technische Universität München, Ismaninger Str. 22, 81675 Munich, Germany Full list of author information is available at the end of the article
}

the insula, rolandic region, and the perisylvian cortex of the dominant hemisphere, still frequently undergo limited debulking or biopsy attributable to the supposed risk of surgery-related deficits [6-9]. Resection of such highly eloquent gliomas always involves a compromise between the extent of resection and the preservation of motor or language function. To achieve both goals, neurosurgeons use multiple modalities to examine, visualize, and monitor anatomy and function presurgically and during resection [10-15]. By carefully choosing a multimodal setup including preoperative mapping of motor and language function

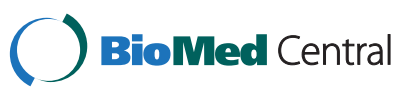


using navigated transcranial magnetic stimulation (nTMS), intraoperative cortical and subcortical mapping using direct cortical stimulation (DCS), intraoperative neuromonitoring (IOM), and awake surgery, we can increase safety and, therefore, allow resection of most such tumors with an acceptable rate of postoperative new deficits [14-23]. Although the literature and data on eloquent glioma surgery are broad, no studies or subgroup analyses are at hand that analyzed the actual functional outcome and oncological benefit of surgery in patients initially diagnosed as inoperable. Thus, we present this retrospective analysis and evaluated all cases that presented to our department for a second opinion. Neurological course, preoperative nTMS, intraoperative DCS mapping, and IOM data were reviewed and related to new postoperative deficits and postoperative imaging. Moreover, clinical outcomes were assessed during follow-up.

\section{Methods}

\section{Patients}

Between 2006 and 2012, we resected 47 out of 51 supratentorial gliomas, which were primarily judged to be non-resectable during prior consultation at another neurosurgical department. These departments were European university departments or at least of university level concerning the range and numbers of surgeries. Four patients with glioma of the basal ganglia did not undergo surgical resection but stereotactic biopsy. During this period between 2006 and 2012, 498 patients underwent surgery of intracranial gliomas in our department.

Decision for surgery was made during an interdisciplinary conference including neurosurgeons, neurooncologists, neuroradiologists, neuropathologists, and radiation oncologists in all cases. An overview of all patients is given in Table 1 . In 9 out of these 47 cases (19\%), the tumor was located within or adjacent to the precentral gyrus, in 15 cases (32\%) within the insula, in 7 cases (15\%) within the postcentral gyrus, in 3 cases (6\%) within the basal ganglia, in 5 cases $(11 \%)$ within the opercular inferior frontal gyrus, in 5 cases $(11 \%)$ within the middle superior temporal gyrus, and in 3 cases (6\%) within the supramarginal gyrus. Mean tumor diameter was $4.9 \pm 2.6 \mathrm{~cm}$ (range $0.4-11.0 \mathrm{~cm}$ ). Tumor size was assessed on T2 FLAIR images for WHO grade II and II and on T1 contrast-enhanced images for WHO grade I and IV. A preoperative motor deficit was present in 13 patients (28\%). Median Karnofsky performance scale (KPS) was 90 (range $40-100 \%$ ). The mean age was $47 \pm 16$ years (range $17-81$ years); 19 patients (40\%) were female and 28 (60\%) were male. Twentyseven tumors (59\%) were in the dominant hemisphere. Indication for awake surgery was a glioma within the left insular and perisylvian region with sufficient remaining language function to perform an intraoperative object naming and counting task. Out of 47 cases, 25 were glioblastomas WHO grade IV (53\%), 14 were anaplastic astrocytomas WHO grade III (30\%), 7 were diffuse astrocytomas WHO grade II (15\%), and one was a pilocytic astrocytoma WHO grade I (2\%). As this report wants to draw attention on the resectability of gliomas per se, we also included this pilocytic astrocytoma in our series because especially these tumors should undergo resection.

Twenty-nine patients (62\%) underwent surgery for recurrent gliomas (grade II: 3 cases; grade III: 9 cases; grade IV: 17 cases). Most common initial symptoms of the patients were seizures in 22 , paresis in 13 , aphasia in 4 , and hemihypesthesia in 2 cases.

\section{Preoperative evaluation}

All patients underwent preoperative magnetic resonance imaging (MRI) for tumor diagnosis, localization, preoperative assessment, and for intraoperative neuronavigation (BrainLAB Vector Vision $2^{\circledR}$ and BrainLAB Curve, BrainLAB AG, Feldkirchen, Germany). Moreover, all patients also received postoperative MR imaging to evaluate the extent of the resection. In addition, every patient was thoroughly examined before and after surgery according to a standardized protocol including handedness, muscle strength, coordination, sensory evaluation, and cranial nerve function. Muscle strength was graded for every muscle in accordance with the British Medical Research Council Scale (BMRC) preoperatively, on the first postoperative day, on the day of discharge, and during postoperative follow-up. Language function was assessed by the Aachen Aphasia Testing Battery preoperatively, at the fifth postoperative day, and 3 and 6 months after surgery [24].

The decision for the use of the different intraoperative techniques such as ultrasound, neuronavigation, fiber tracking, MEP monitoring, or awake surgery was done by the operating surgeon depending on the specific tumor location.

\section{Anesthesia}

As volatile anesthetics have been shown to severely interfere with IOM, we used total intravenous anesthesia in all cases without exception and strictly avoided the use of volatile anesthetics before and during surgery [25-27]. Thus, anesthesia was induced and maintained by continuous propofol administration, and intraoperative analgesia was achieved through continuous administration of remifentanyl. Neuromuscular blocking was avoided during surgery and only used for intubation by rocuronium. 
Table 1 Patient characteristics

\begin{tabular}{|c|c|c|c|c|c|c|c|c|c|c|c|c|c|}
\hline $\mathrm{Pt} \#$ & $\begin{array}{l}\text { WHO } \\
\text { grade }\end{array}$ & $\begin{array}{l}\text { Recurrent } \\
\text { tumor }\end{array}$ & $\begin{array}{l}\text { Tumor } \\
\text { diameter }\end{array}$ & $\begin{array}{c}\text { Preop } \\
\text { TMZ }\end{array}$ & $\begin{array}{l}\text { Preop } \\
\text { RTx }\end{array}$ & $\begin{array}{l}\text { Preop } \\
\text { motor } \\
\text { deficit }\end{array}$ & $\begin{array}{l}\text { Postop } \\
\text { motor } \\
\text { deficit }\end{array}$ & $\begin{array}{c}\text { Preop } \\
\text { language } \\
\text { deficit }\end{array}$ & $\begin{array}{c}\text { Postop } \\
\text { language } \\
\text { deficit }\end{array}$ & $\begin{array}{l}\text { Preop } \\
\text { KPS }\end{array}$ & $\begin{array}{l}\text { Postop } \\
\text { KPS }\end{array}$ & EOR & $\begin{array}{l}\text { Awake } \\
\text { surgery }\end{array}$ \\
\hline 1 & III & $Y$ & 11.0 & $Y$ & $Y$ & $\mathrm{~N}$ & $\mathrm{~N}$ & $\mathrm{~N}$ & $\mathrm{~N}$ & 90 & 90 & GTR & $N$ \\
\hline 2 & III & $\mathrm{N}$ & 5.1 & $\mathrm{~N}$ & $N$ & $\mathrm{~N}$ & $\mathrm{~T}$ & $N$ & $\mathrm{~T}$ & 70 & 60 & GTR & $Y$ \\
\hline 3 & III & $Y$ & 6.8 & $Y$ & $\mathrm{~N}$ & $Y$ & $\mathrm{~N}$ & $\mathrm{~N}$ & $\mathrm{~N}$ & 70 & 70 & GTR & $N$ \\
\hline 4 & III & $Y$ & 10.8 & $\mathrm{~N}$ & $\mathrm{~N}$ & $\mathrm{~N}$ & $\mathrm{~T}$ & $N$ & $\mathrm{~N}$ & 90 & 70 & GTR & $N$ \\
\hline 5 & IV & $Y$ & 9.2 & $Y$ & $\mathrm{~N}$ & $Y$ & $N$ & $N$ & $\mathrm{~T}$ & 80 & 80 & STR & $Y$ \\
\hline 6 & IV & $\mathrm{N}$ & 4.5 & $\mathrm{~N}$ & $\mathrm{~N}$ & $\mathrm{~N}$ & $\mathrm{~N}$ & $N$ & $\mathrm{~T}$ & 70 & 70 & GTR & $Y$ \\
\hline 7 & IV & $Y$ & 1.9 & $Y$ & $Y$ & $\mathrm{~N}$ & $\mathrm{~T}$ & $\mathrm{~N}$ & $\mathrm{~N}$ & 80 & 60 & STR & $\mathrm{N}$ \\
\hline 8 & IV & $Y$ & 0.4 & $Y$ & $Y$ & $\mathrm{~N}$ & $N$ & $N$ & $\mathrm{~N}$ & 100 & 100 & STR & $N$ \\
\hline 9 & IV & Y & 4.5 & $Y$ & $Y$ & $Y$ & $\mathrm{~T}$ & $\mathrm{~N}$ & $\mathrm{~N}$ & 90 & 90 & GTR & $\mathrm{N}$ \\
\hline 10 & IV & $\mathrm{N}$ & 5.6 & Y & $\mathrm{N}$ & $\mathrm{N}$ & $N$ & $\mathrm{~N}$ & $\mathrm{~N}$ & 90 & 90 & GTR & $N$ \\
\hline 11 & $\|$ & $Y$ & 5.1 & $\mathrm{~N}$ & $\mathrm{~N}$ & $\mathrm{~N}$ & $N$ & $\mathrm{~N}$ & $\mathrm{~N}$ & 90 & 90 & GTR & $Y$ \\
\hline 12 & III & $Y$ & 4.5 & $Y$ & $\mathrm{~N}$ & $\mathrm{~N}$ & $\mathrm{~N}$ & $N$ & $\mathrm{~N}$ & 100 & 100 & GTR & $\mathrm{N}$ \\
\hline 13 & $\|$ & $\mathrm{N}$ & 7.9 & $Y$ & $\mathrm{~N}$ & $Y$ & $\mathrm{~T}$ & $N$ & $\mathrm{~T}$ & 70 & 70 & GTR & Y \\
\hline 14 & $\|$ & $\mathrm{N}$ & 6.1 & $\mathrm{~N}$ & $\mathrm{~N}$ & $\mathrm{~N}$ & $\mathrm{~N}$ & $\mathrm{~N}$ & $\mathrm{~N}$ & 100 & 100 & GTR & $\mathrm{N}$ \\
\hline 15 & IV & $Y$ & 3.0 & $Y$ & $Y$ & $\mathrm{~N}$ & $\mathrm{~N}$ & $N$ & $\mathrm{~N}$ & 100 & 100 & GTR & $\mathrm{N}$ \\
\hline 16 & III & $Y$ & 6.4 & $\mathrm{~N}$ & $\mathrm{~N}$ & $\mathrm{~N}$ & $N$ & $\mathrm{~N}$ & $\mathrm{~N}$ & 100 & 100 & GTR & $N$ \\
\hline 17 & IV & $Y$ & 7.0 & $Y$ & $Y$ & $\mathrm{~N}$ & $\mathrm{~T}$ & $\mathrm{~N}$ & $\mathrm{~N}$ & 70 & 30 & GTR & $\mathrm{N}$ \\
\hline 18 & III & Y & 0.7 & Y & $\mathrm{N}$ & $N$ & $\mathrm{~T}$ & $N$ & $\mathrm{~N}$ & 100 & 80 & GTR & $\mathrm{N}$ \\
\hline 19 & III & $\mathrm{N}$ & 9.3 & $\mathrm{~N}$ & $Y$ & $\mathrm{~N}$ & $N$ & $N$ & $\mathrm{~N}$ & 100 & 100 & GTR & $N$ \\
\hline 20 & $\|$ & $Y$ & 4.6 & $Y$ & $\mathrm{~N}$ & $\mathrm{~N}$ & $\mathrm{~T}$ & $\mathrm{~N}$ & $T$ & 100 & 90 & GTR & $Y$ \\
\hline 21 & IV & $Y$ & 2.6 & $Y$ & $Y$ & $\mathrm{~N}$ & $\mathrm{~T}$ & $\mathrm{~N}$ & $\mathrm{~N}$ & 50 & 50 & GTR & $\mathrm{N}$ \\
\hline 22 & IV & $Y$ & 2.0 & $Y$ & Seed & $Y$ & $P$ & $N$ & $P$ & 80 & 70 & STR & $Y$ \\
\hline 23 & IV & $Y$ & 4.9 & $Y$ & $Y$ & $\mathrm{~N}$ & $N$ & $\mathrm{~N}$ & $\mathrm{~N}$ & 90 & 80 & GTR & $\mathrm{N}$ \\
\hline 24 & IV & $\mathrm{N}$ & 6.8 & $Y$ & $\mathrm{~N}$ & $Y$ & $N$ & $N$ & $\mathrm{~N}$ & 70 & 70 & STR & $N$ \\
\hline 25 & $\|$ & $\mathrm{N}$ & 5.6 & $\mathrm{~N}$ & $\mathrm{~N}$ & $\mathrm{~N}$ & $N$ & $\mathrm{~N}$ & $\mathrm{~N}$ & 70 & 70 & STR & $\mathrm{N}$ \\
\hline 26 & IV & $Y$ & 2.9 & $Y$ & $Y$ & $Y$ & $\mathrm{~N}$ & $\mathrm{~N}$ & $\mathrm{~N}$ & 70 & 70 & GTR & $N$ \\
\hline 27 & IV & $N$ & 4.6 & $\mathrm{~N}$ & $\mathrm{~N}$ & $N$ & $N$ & $N$ & $\mathrm{~N}$ & 90 & 90 & GTR & $N$ \\
\hline 28 & III & $Y$ & 4.0 & $Y$ & $N$ & $Y$ & P & $N$ & $\mathrm{~N}$ & 90 & 50 & GTR & $N$ \\
\hline 29 & IV & $Y$ & 6.0 & $Y$ & $Y$ & $\mathrm{~N}$ & $N$ & $\mathrm{~N}$ & $\mathrm{~N}$ & 80 & 80 & GTR & $\mathrm{N}$ \\
\hline 30 & III & $\mathrm{N}$ & 10.0 & $Y$ & $\mathrm{~N}$ & $N$ & $\mathrm{~N}$ & $N$ & $\mathrm{~N}$ & 90 & 90 & STR & $\mathrm{N}$ \\
\hline 31 & III & $\mathrm{N}$ & 5.3 & $Y$ & $Y$ & $\mathrm{~N}$ & $\mathrm{~N}$ & $\mathrm{~N}$ & $\mathrm{~N}$ & 90 & 90 & GTR & $\mathrm{N}$ \\
\hline 32 & IV & $\mathrm{N}$ & 3.3 & $\mathrm{~N}$ & $Y$ & $Y$ & $N$ & $\mathrm{~N}$ & $\mathrm{~N}$ & 40 & 40 & STR & $\mathrm{N}$ \\
\hline 33 & III & $\mathrm{N}$ & 4.0 & $Y$ & $\mathrm{~N}$ & $\mathrm{~N}$ & $N$ & $\mathrm{~N}$ & $\mathrm{~T}$ & 90 & 90 & STR & $Y$ \\
\hline 34 & $\|$ & $Y$ & 1.5 & $\mathrm{~N}$ & $N$ & $\mathrm{~N}$ & $N$ & $N$ & $\mathrm{~N}$ & 100 & 100 & GTR & $N$ \\
\hline 35 & III & $Y$ & 1.1 & $Y$ & $\mathrm{~N}$ & $N$ & P & $N$ & $\mathrm{~N}$ & 90 & 40 & GTR & $\mathrm{N}$ \\
\hline 36 & IV & $Y$ & 6.0 & $\mathrm{~N}$ & $\mathrm{~N}$ & $\mathrm{~N}$ & $N$ & $N$ & $\mathrm{~N}$ & 90 & 90 & GTR & $N$ \\
\hline 37 & IV & $Y$ & 1.4 & $Y$ & $Y$ & $\mathrm{~N}$ & $\mathrm{~T}$ & $\mathrm{~N}$ & $N$ & 100 & 50 & GTR & $\mathrm{N}$ \\
\hline 38 & III & $Y$ & 7.1 & $Y$ & $\mathrm{~N}$ & $\mathrm{~N}$ & $\mathrm{~T}$ & $\mathrm{~N}$ & $\mathrm{~N}$ & 100 & 60 & STR & $\mathrm{N}$ \\
\hline 39 & 1 & $N$ & 1.5 & $\mathrm{~N}$ & $\mathrm{~N}$ & Y & $N$ & $N$ & $\mathrm{~N}$ & 50 & 100 & GTR & $N$ \\
\hline 40 & IV & Y & 2.4 & $Y$ & $Y$ & Y & $\mathrm{T}$ & $N$ & $\mathrm{~N}$ & 60 & 50 & GTR & $N$ \\
\hline 41 & IV & $\mathrm{N}$ & 6.0 & $Y$ & $Y$ & $\mathrm{~N}$ & $N$ & $N$ & $\mathrm{~N}$ & 100 & 100 & GTR & $N$ \\
\hline 42 & IV & $Y$ & 5.6 & $Y$ & $Y$ & $\mathrm{~N}$ & $\mathrm{~N}$ & $\mathrm{~N}$ & $\mathrm{~N}$ & 90 & 90 & GTR & $\mathrm{N}$ \\
\hline 43 & IV & $\mathrm{N}$ & 4.5 & $Y$ & $Y$ & $Y$ & $N$ & $N$ & $N$ & 50 & 50 & GTR & $\mathrm{N}$ \\
\hline
\end{tabular}


Table 1 Patient characteristics (Continued)

\begin{tabular}{|c|c|c|c|c|c|c|c|c|c|c|c|c|c|}
\hline 44 & IV & $Y$ & 4.9 & $Y$ & $Y$ & $\mathrm{~N}$ & $P$ & $\mathrm{~N}$ & $\mathrm{~N}$ & 90 & 80 & GTR & $\mathrm{N}$ \\
\hline 45 & IV & $Y$ & 5.0 & $Y$ & $Y$ & $\mathrm{~N}$ & $\mathrm{~N}$ & $\mathrm{~N}$ & $\mathrm{~N}$ & 90 & 90 & GTR & $N$ \\
\hline 46 & IV & $\mathrm{N}$ & 4.5 & $Y$ & $Y$ & $Y$ & $\mathrm{~T}$ & $\mathrm{~N}$ & $\mathrm{~N}$ & 80 & 70 & STR & $\mathrm{N}$ \\
\hline 47 & $\|$ & $\mathrm{N}$ & 2.0 & $Y$ & $\mathrm{~N}$ & $\mathrm{~N}$ & $\mathrm{~T}$ & $\mathrm{~N}$ & $\mathrm{~N}$ & 100 & 80 & STR & $\mathrm{N}$ \\
\hline
\end{tabular}

Patient characteristics of the 47 patients, which underwent surgical resection. Tumor diameter (in cm), preoperative deficit, postoperative deficit $(T=$ temporary, $\mathrm{P}=$ permanent, $\mathrm{N}=$ no deficit), and Karnofsky Performance Scale (KPS) are outlined. $\mathrm{Y}=\mathrm{yes}, \mathrm{N}=$ no. TMZ $=$ Temozolomide. $\mathrm{RTx}=$ radiotherapy. EOR = extent of resection. $\mathrm{STR}=$ subtotal resection. $\mathrm{GTR}=$ gross total resection .

\section{Neuronavigation}

Positron emission tomography (PET) images were fused with continuous sagittal images of T1-weighted 3D gradient echo sequence, T2 FLAIR, and DTI data. In 11 patients (23\%), nTMS was also used to map cortical language and motor areas preoperatively; nTMS data were then fused into the neuronavigation dataset. Finally, data were transmitted to the neuronavigation system (BrainLAB Vector Vision $2^{\circledR}$ and BrainLAB Curve $^{\circledR}$, BrainLAB AG, Feldkirchen, Germany), as previously described $[13,14]$.

\section{Intraoperative MEP monitoring}

IOM by direct cortical stimulation was used in 38 of 47 cases (81\%). Subsequent to craniotomy and durotomy, a strip electrode with eight contacts $\left(\mathrm{ADTech}^{\circledR}\right.$ strip electrode, AD Technic, City, WI, USA or Inomed Medizintechnik, Emmendingen, Germany) was positioned subdurally onto the cortex of the rolandic region. An angle of $60-70^{\circ}$ to the supposed central sulcus was aimed at. After positioning the strip electrode, the median nerve was stimulated and the central sulcus was identified by somatosensory evoked potential phase reversal [28]. DCS mapping of the motor cortex was then performed with intensities between 5 and $14 \mathrm{~mA}$, square-wave pulse with duration of $0.2-0.3 \mathrm{~ms}$, frequency of $350 \mathrm{~Hz}$, and a train of 5 pulses as previously reported $[15,28,29]$. To stimulate motor evoked potential (MEP) monitoring of the upper and lower extremity, square-wave pulses with duration of 200-700 $\mu \mathrm{s}$, a frequency of $350 \mathrm{~Hz}$, and a train of 5 pulses were applied. The used protocol was published previously [15]. Decline in amplitude of more than 50\%, which was not explained by technical issues, was considered a considerable deterioration and was reported to the surgeon. If changes of compound muscle action potential (CMAP) occurred, the event was instantly reported to the neurosurgeon, who reversed the causative maneuver, if possible. Partial loss of CMAP from related muscle groups was regarded as a decline rather than a loss. Latency increases devoid of concomitant deterioration of amplitude rarely occurred.

\section{Awake monitoring}

Awake surgery was only performed when the tumor was within the left insula, operculum, dorsal superior temporal gyrus, angular gyrus, and supramarginal gyrus. Tumors within the left pre- or postcentral gyrus were not operated by awake surgery. The day before surgery, a neuropsychologist trained the patient for the object naming task and baseline testing of all pictures was performed. Only pictures that were named fluently were included for intraoperative mapping. In surgery, the patient was positioned supine and $45^{\circ}$ to the right side. Before sharp fixation of the head, regional anesthesia was applied to the galea by bupivacaine. Fifteen minutes before language mapping, propofol infusion was stopped and remifentanyl was progressively reduced to achieve an optimum level of analgesia during mapping. DCS mapping was performed using bipolar stimulation every $5 \mathrm{~mm}$ using 3 to $15 \mathrm{~mA}$ over 4 seconds and a $60 \mathrm{~Hz}$ technique. To detect afterdischarges, a direct cortical electroencephalogram was recorded with 8 channels. During mapping, pictures of common objects were presented to the patient in a time-locked way, and elicited speech impairment was evaluated by the neuropsychologist. The patient had to name the object and start every naming with the sentence "This is..." Positive sites were marked at the cortical surface with numbers indicating the evoked disturbance. After completion of cortical mapping, the resection was performed under continuous language testing to also monitor affection of subcortical fiber tracts. After resection, the patient was then sedated during wound closure.

\section{Tumor resection}

An ultrasound aspirator (Sonopet Ultrasonic Aspirator, Stryker Medical, Portage, MI, USA) as well as neuronavigation was used for all cases. Upon any amplitude loss or decline of more than $50 \%$ of the initial MEP amplitude in at least one channel, resection was halted, spatulas removed, and the surgical field was irrigated with warm Ringer's solution. The MEP technique is extensively described above (Intraoperative MEP monitoring). In cases of awake surgery, resection was immediately stopped whenever the neuropsychologist reported deterioration of language function. In cases of resection close to a major vasculature, the surgical field was irrigated with nimodipine to reverse potential vasospasm. After renormalization/stabilization of MEPs, resection was continued. If potentials did not recover, resection was stopped at this tumor region. 


\section{Postoperative evaluation}

For every patient, neurological status was directly assessed after surgery, 6-8 weeks postoperatively and during follow-up on a regular basis every 3-12 months, depending on the tumor entity. Moreover, each patient underwent an MRI scan within 48 hours after operation. During follow-up, MRI scans were also performed every 3-12 months depending on the tumor grade. Thus, we evaluated the MRI scan of the first postoperative day with regard to the extent of the resection, increasing edema, diffusion impairment, and bleeding to find explanations for neurological deterioration without intraoperatively MEP changes. Extent of resection was defined as gross total resection (GTR) or subtotal resection depending on the presence of residual tumor on T2 FLAIR (WHO grade II and III) or T1 contrast-enhanced sequences (WHO grade I and IV). Furthermore, we evaluated every MRI scan during follow-up for recurrent tumors. Neurological status in this study was only considered during progression-free survival. New postoperative neurological motor deficit was distinguished between temporary and permanent deficit. Temporary deficit was defined as a new or aggravated postoperative motor deficit that disappeared at least until the 6- to 8-week follow-up. Permanent deficit was defined as new or aggravated postoperative motor deficit that did not resolve during follow-up.

\section{Ethical standard}

The study is well in accordance with the ethical standards of the Technical University of Munich, the local ethics committee (registration number: 2826/10), and the Declaration of Helsinki.

\section{Statistical analysis}

To test the distribution of several attributes, a chi-square or Fisher Exact test was performed. Differences between groups were tested using the Kruskall-Wallis test for nonparametric one-way analysis of variance (ANOVA), followed by Dunn's test as the post hoc test. Differences between two groups were tested using the Mann-WhitneyWilcoxon test for multiple comparisons on ranks for independent samples, followed by Dunn's test as the post hoc test. All results are presented as mean \pm standard deviation (SD). Median and range were also delivered (GraphPad Prism 5.0 c, La Jolla, CA, USA); p $<0.05$ was considered significant.

\section{Results}

GTR was achieved in 35 cases (74\%) (Figure 1). Awake surgery was performed in 8 cases $(17 \%)$, whereas 38 cases (81\%) were performed under continuous MEP monitoring. Three cases (6\%) received awake craniotomy and MEP monitoring for subcortical dissection within the pyramidal tract after the awake phase. Thus, 4 cases underwent surgery without MEP or awake monitoring. For evaluation and follow-up of neurological function, we only considered neurological status during progression-free survival, which was 11.3 months (range: 2 weeks - 64.5 months) and median overall survival was 14.8 months (range: 4 weeks 20.5 months) depending on recurrence and malignancy (Table 2). Before surgery, not only recurrent but also some newly diagnosed gliomas were treated using chemo- or radiotherapy. Table 3 provides an overview. Moreover, there were no healing problems or postoperative infections in the patients within this cohort.

\section{Preoperative functional mapping}

Navigated TMS was used for preoperative mapping of language areas in 4 cases and motor areas in 6 cases because 2 cases underwent combined motor and language mapping.

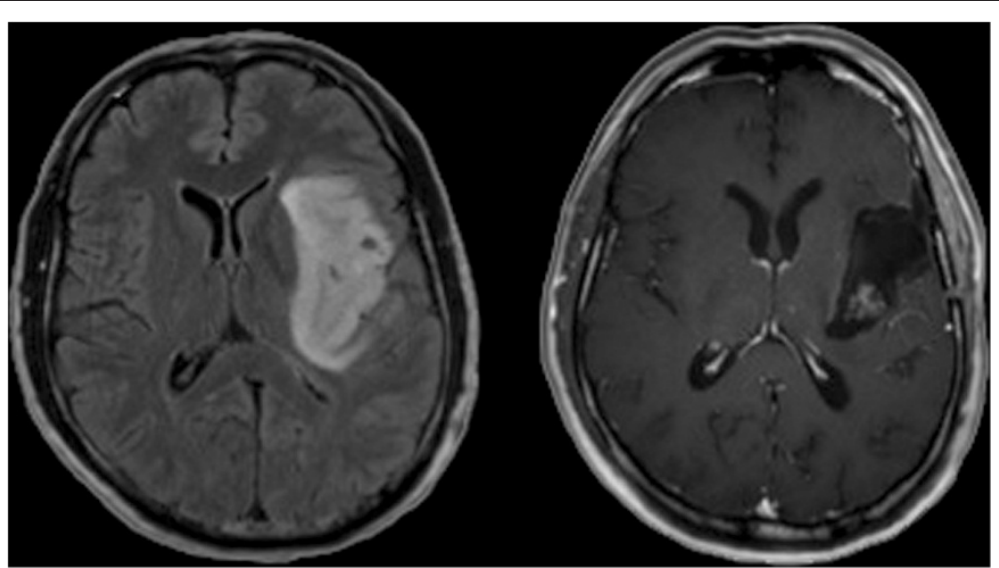

Figure 1 Illustrative case of gross total resection of a left-sided insular glioma WHO grade 3. 
Table 2 Follow-up and overall survival

\begin{tabular}{lcccccc}
\hline & \multicolumn{2}{c}{$\begin{array}{c}\text { mean follow-up } \\
\text { (months) }\end{array}$} & & & \multicolumn{2}{c}{$\begin{array}{c}\text { mean overall survival } \\
\text { (months) }\end{array}$} \\
\cline { 2 - 3 } \cline { 6 - 7 } & primary & recurrent & & primary & recurrent \\
\hline WHO grade I & 47.9 & - & & alive & - \\
WHO grade II & 38.3 & 26.0 & & all alive & all alive \\
WHO grade III & 21.6 & 22.0 & & all alive & 20.5 \\
WHO grade IV & 8.6 & 7.9 & & 5.1 & 6.0 \\
\hline
\end{tabular}

Columns 2 \& 3: mean follow-up for alive patients. Columns 4 \& 5: overall survival of deceased patients. This series only contains one patient with initially diagnosed WHO grade I glioma. When patients are alive, mean overall survival equals to mean follow-up.

\section{Further used modalities}

Neuronavigation was applied in all cases. Diffusion tensor imaging fiber tracking was included in 18 (38\%); fluorescence guidance using 5-aminolevulinic acid was applied in 18 (38\%); and intraoperative ultrasound was used in 1 case.

\section{Awake craniotomy}

Of patients undergoing awake surgery, 5 patients $(63 \%)$ suffered from initially diagnosed and 3 patients (37\%) suffered from recurrent glioma. After awake craniotomy on 8 patients, 6 patients (75\%) showed a new aphasia at the first postoperative day but only 1 patient (13\%) experienced a permanent surgery-related aggravated aphasia during long-term follow-up. GTR was possible in 5 cases $(63 \%)$.

\section{Correlation of tumor type and location to postoperative motor deficit}

Postoperative temporary or permanent impairment of motor function was significantly higher in recurrent tumors: After primary glioma resection (18 patients), no patients showed any permanent deficit, whereas 4 patients $(22 \%)$ presented with temporary and 14 patients (78\%) with no new postoperative motor deficit. However, after resection of recurrent glioma (28 patients), 4 patients (14\%) showed permanent and 10 patients (34\%) showed temporary surgery-related new paresis. Thus, 15 patients (52\%) showed no new motor deficit (Figure 2).

Table 3 Presurgical therapy

\begin{tabular}{lccccc}
\hline & \multicolumn{2}{c}{ Primary surgery } & & \multicolumn{2}{c}{ Recurrent tumor } \\
\cline { 2 - 3 } & cases & $\%$ & & cases & $\%$ \\
\hline RTx only & 2 & 11 & & 0 & 0 \\
TMZ only & 6 & 33 & & 8 & 28 \\
TMZ + RTx & 0 & 0 & 2 & 7 \\
Seed & 0 & 0 & 1 & 3
\end{tabular}

An overview on presurgical chemo- or radiotherapy in patients with recurrent but also with initially diagnosed gliomas, after which non-resectability was noted. Temozolomide (TMZ) and radiotherapy (RTx) were also applied combined.
As expected, postoperative temporary and permanent impairment of motor function were related to tumor location with no respect to initial or recurrent tumor. After resection of gliomas in the precentral gyrus, 11\% of all patients (1 patients) experienced permanent deterioration of motor function. Additionally, 44\% of patients (4 patients) with a precentral glioma showed a temporary motor function deficit. After resection of insular gliomas, patients showed temporary deficit in $33 \%$ (5 patients) and permanent deficit in $7 \%$ of all cases (1 patient). Patients with gliomas affecting the subcortical white matter temporarily deteriorated in $67 \%$ (2 patients) and permanently deteriorated in $33 \%$ (1 patient) of cases with regard to motor function.

\section{MEP monitoring}

In all intended 38 cases, IOM through continuous MEP monitoring was possible. MEPs were stable throughout the operation in 18 patients (47\%), showed reversible amplitude decline of more than $50 \%$ baseline but recovered in 15 patients (39\%), and irreversible amplitude declined more than $50 \%$ baseline in 5 patients (13\%). Postoperatively, 18 patients (39\%) had a new motor deficit, which remained permanent in 4 patients (8.5\%). Irreversible MEP decline was only observed in WHO grade III and grade IV gliomas, but no other significant difference existed with respect to the different tumor types (data not shown). Out of those 20 cases (52\%) with MEP amplitude decline, resection was temporarily stopped, attributable to IOM in 10 cases (26\% of all 38 IOM cases) and completely halted in 6 of these cases (16\% of all 38 IOM cases). Immediately after MEP decline, retractors were repositioned and the resection cavity was additionally irrigated. In 5 of these 10 cases (50\%), STR was achieved, whereas STR was performed in only 3 out of 28 cases (11\%), which were not influenced by IOM due to stable amplitudes $(\mathrm{p}=0.0186$; Figure 3$)$. Postoperative new temporary or permanent motor deficits were similar in the STR (unchanged: $58 \%$, temporary: 33\%, permanent: $9 \%$ of 12 cases) and GTR groups (unchanged: 63\%, temporary: 28\%, permanent: $9 \%$ of 35 cases) (Figure 4). In contrast, in those 10 cases in which the surgeon had to stop resection because of considerable MEP decline, we recognized an unchanged motor function in 30\% of cases and a new temporary deficit in $60 \%$ of cases, and new permanent motor deficit in $10 \%$ of cases. Without the influence of IOM, motor function was unchanged in $68 \%$ of cases, temporarily deteriorated in $21 \%$ of cases, and permanently deteriorated in $11 \%$ of cases $(p=0.07$; Figure 5). Although the data failed to show statistical significance, they showed a trend toward a higher rate of temporary motor deficit in patients in which resection was limited by IOM. 


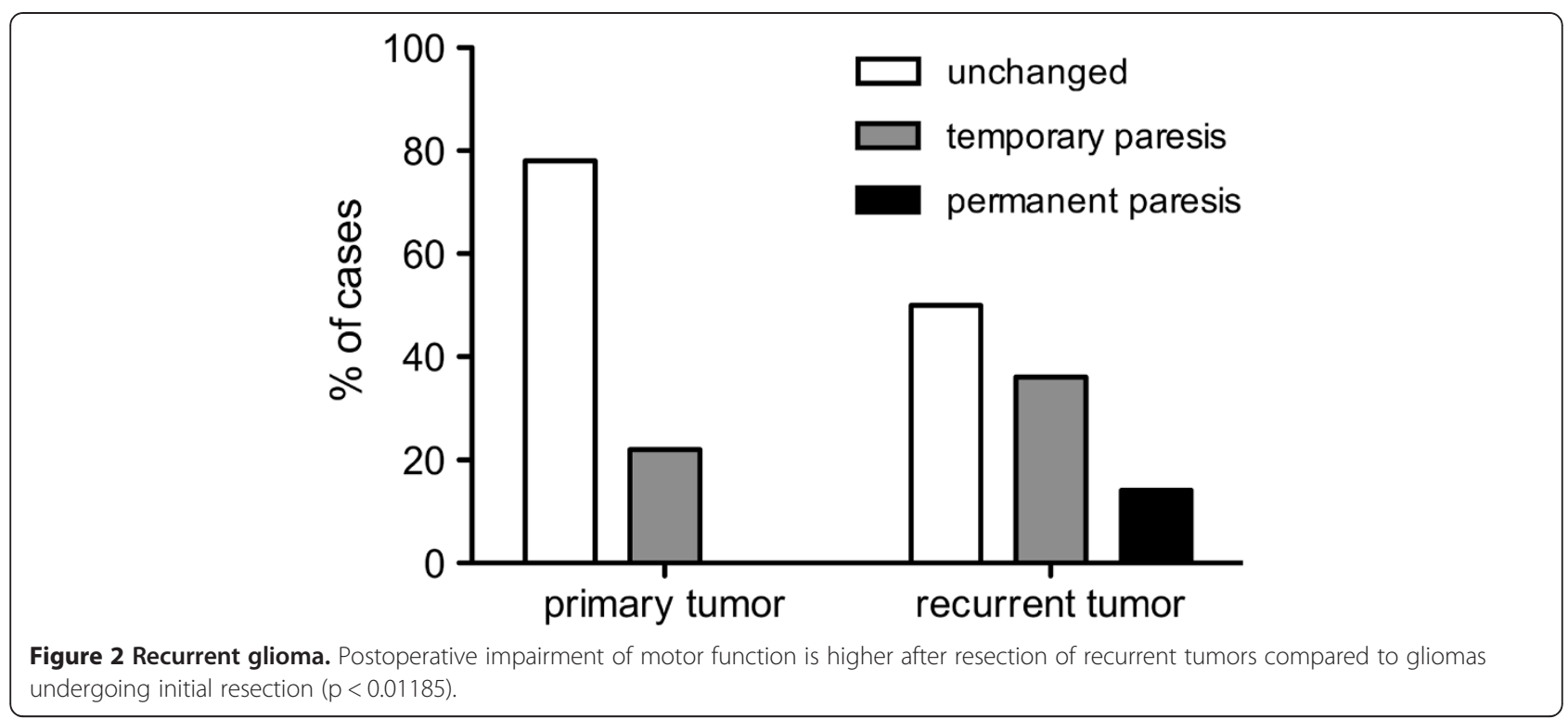

\section{Postoperative MRI scans}

To find sufficient basis for the explanation of postoperative neurological deterioration, we evaluated all postoperative MRI scans. Nine patients (13\%) had temporary new motor deficit despite recovered MEP decline in which MRI revealed increasing edema in 4 cases and secondary hemorrhage within the resection cavity in 5 cases. However, only 3 out of these 5 cases were symptomatic and underwent revision surgery at the same day. Out of those 4 patients with new permanent surgery-related paresis, 2 presented with ischemic lesions at the border of the resection cavity and 2 showed resection within motor eloquent regions. With regard to the 8 awake cases, 2 patients showed temporarily and 1 patient

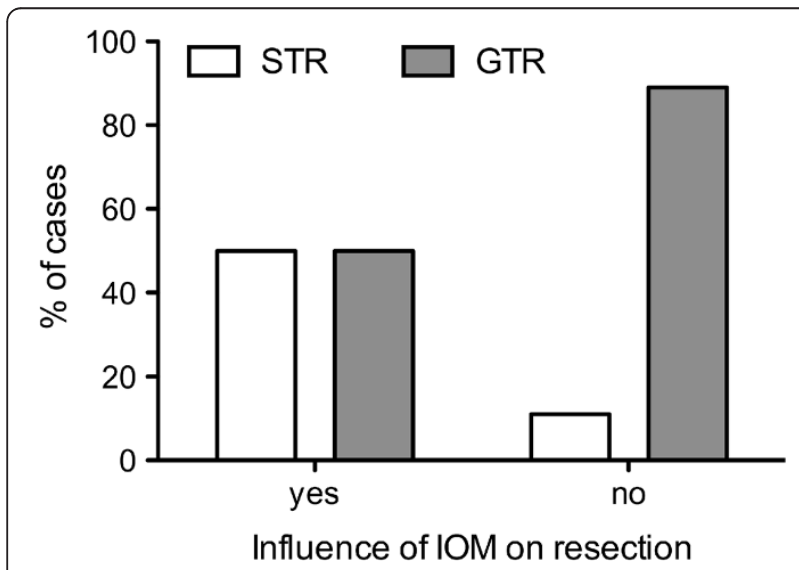

Figure 3 Influence of IOM on the extent of resection. When surgery was influenced by IOM due to MEP amplitude decline of more than $50 \%$ baseline, gross total resection (GTR) was only achieved in 50\% of cases, whereas GTR was achieved in $89 \%$ of cases in which IOM showed no impact on surgery due to stable amplitudes (p0.0186) presented with permanently deteriorated language function. All 3 cases were glioblastoma multiforme within the angular gyrus and postoperative MRI showed no edema, hemorrhage, or ischemia.

\section{Operation on recurrent gliomas}

In this series, we operated on 29 recurrent gliomas. Three were WHO grade II, 9 were WHO grade III, and 17 were glioblastoma (GBM). Of these patients, 7 (24\%) already had preoperative paresis. Four patients were operated awake and one of these patients (25\%) suffered from preoperative aphasia. However, continuous MEP monitoring was possible in all 24 intended cases (83\%). Compared with the first operation, resection of recurrent gliomas showed a lower degree of subtotal resections but without reaching statistical significance (17\% in recurrent and $39 \%$ in the first operation). Concerning resections of recurrent glioma, postoperative new permanent deficits were observed in $14 \%$ of all cases (4 patients) (aphasia: 3\%, paresis: 11\%), whereas temporary deficits occurred in $35 \%$ of cases (10 cases) (aphasia: 10\%, paresis: 25\%) (Figure 2). Pre- as well as postoperative KPS was also comparable in patients who underwent the first (before: 85, after surgery: 90) and repeated resection (before: 85 , after surgery: 80 ).

\section{Discussion}

During the last decade, surgical resection became increasingly important as part of a multimodal therapeutic regime for the treatment of high- and low-grade gliomas $[1,4,23,30,31]$. However, even today, many gliomas within highly eloquent cortical regions still regularly undergo only debulking or biopsy. The most striking argument for this approach is the risk of surgery-related 


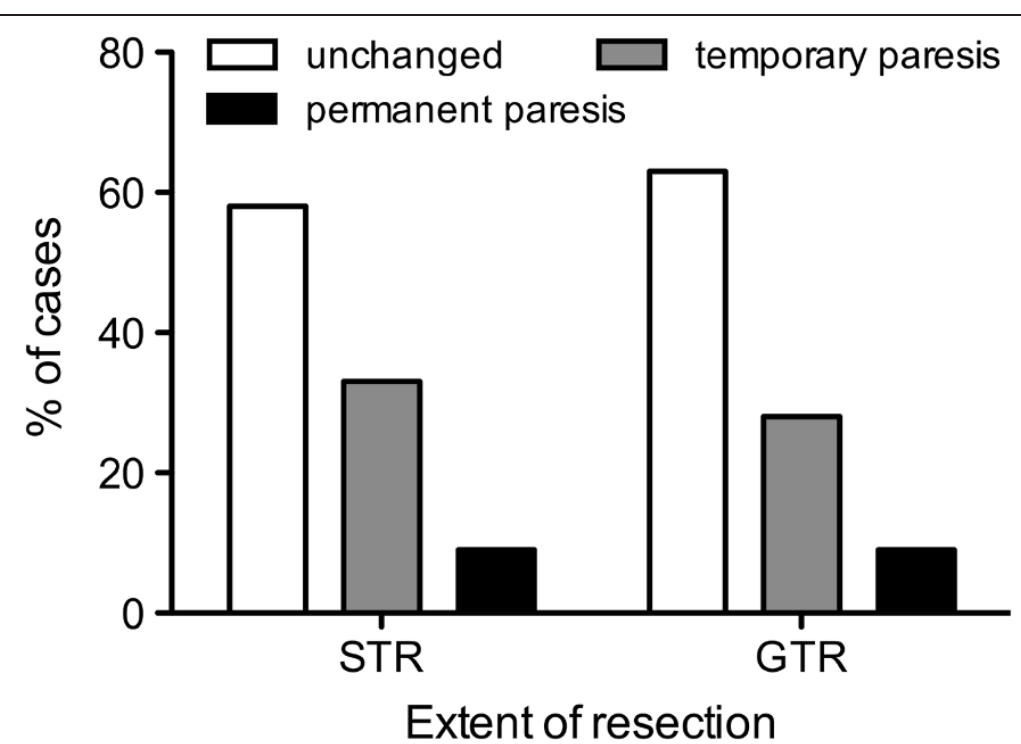

Figure 4 Extent of resection vs. postoperative paresis. Postoperative new temporary or permanent motor deficits were highly comparable in patients with subtotal (STR) and gross total (GTR) resection.

deficits [6-9]. Nonetheless, with regard to already published data on surgery on eloquent gliomas, the risk of new neurological deficits seems moderate [15,18,22,23,31-33]. Especially when a multimodal and function-guided approach is used [34]. Yet, no studies or subgroup analyses exist that reviewed the actual functional outcomes and oncological benefits of surgery in patients initially diagnosed as inoperable.

In our series, only $8.5 \%$ of all patients with gliomas in or adjacent to eloquent motor areas suffered from new permanent deterioration of motor function after surgery (Figure 2). Regarding these data, our study is well in accordance with previous studies $[26,35,36]$. When also considering the high postoperative KPS in initially diagnosed and recurrent gliomas, we have to strongly reject the argument that these patients have an unacceptable high risk of surgery-related disability or loss in quality of life.

With regard to the GBM subgroup, median survival was comparable to the non-surgical series; however, KPS was higher in our patients even after surgery. Thus, high-quality survival was improved (Table 2) [7-9,37].

Concerning the impact of the extent of resection on the actual survival the subgroups of this study are to

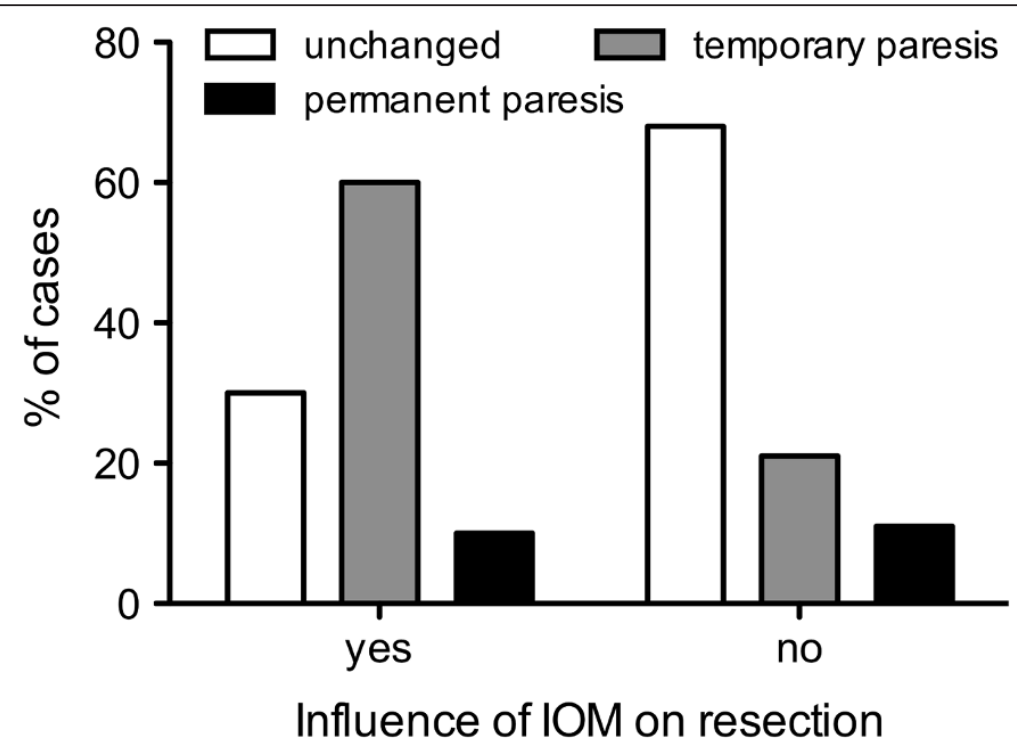

Figure 5 IOM vs. postoperative paresis. When surgery was influenced by IOM due to MEP amplitude decline of more than $50 \%$ baseline data showed a trend towards a higher rate of temporary motor deficit compared to patients in which resection was not affected by IOM ( $\mathrm{p}=0.07$ ). 
small for such a statistical analysis. Thus, this study has to be considered as a pilot study.

\section{Preoperative functional mapping}

As also reported previously, we observed an important impact from preoperative nTMS mapping of the motor eloquent cortex $[14,38]$. Moreover, 4 additional patients underwent nTMS mapping of the language eloquent cortex. Although nTMS language mapping still requires further research, it is already a valuable tool in a multimodal approach $[39,40]$.

\section{Correlation of tumor type and location to postoperative motor deficit}

In our series, most tumors were located within the insula, rolandic region, or the perisylvian cortex. When analyzing our data, we were not able to show any statistically significant difference for the risk of surgeryrelated new motor deficit with regard to tumor location. Thus, we cannot identify any of these structures to be less eligible for surgical resection, which is well in accordance with previous findings [15]. However, we must emphasize that surgery of recurrent glioma has a significantly higher risk of surgery-related new motor deficit (Figure 2), which was also found by others and has to be kept in mind when advising our patients $[15,41]$. The reasons for this phenomenon are supposed to be primarily vascular. As primary resection of these gliomas usually reaches the borders of motor or language eloquent regions, recurrent tumor growth invades this eloquent brain tissue and its supplying arteries. Thus, our series showed that surgery of recurrent gliomas causes a higher rate of ischemia adjacent to the resection cavity as initial surgery does, which is contradictive to previous studies [41]. Moreover, chemotherapy as well as radiation therapy might alter neuronal and vascular metabolism and therefore impair motor plasticity as it has been described recently [42].

\section{MEP monitoring}

MEP amplitude decline caused a significantly higher rate of STR (Figure 3). However, this group also showed a lower rate of temporary but not of permanent new motor deficits (Figure 4). However, this result seems to mostly come from the small number of cases (10 patients) in which surgery was influenced by IOM. Without the influence of IOM on motor function, we failed to show statistical significance. However, the data showed a trend toward a higher rate of temporary motor deficit in patients in which resection was limited by IOM. Yet, the rate of permanent motor deficits was identical (Figure 5). These findings have to be interpreted as a result of the small group of patients with influence of IOM on the course of surgery (10 patients) because larger series indeed showed an influence of IOM on the functional outcome of long-term follow-up [15,17].

Concerning those 2 cases of reversible MEP decline with permanently new motor deficit, in which we observed partial removal of the primary motor cortex we have to state that this partial resection of rolandic cortex is not the only explanation although it is the only explanation, which can be observed on postoperative MRI. A dislocation of the cortical MEP electrode and replacement to another cortical muscle representation is also an explanation that has to be mentioned.

\section{Recurrent gliomas}

In this series, we operated on 29 recurrent gliomas. Compared with the first operation, resection of recurrent gliomas showed a surprisingly lower degree of STR but without reaching statistical significance $(17 \%$ in recurrent and $39 \%$ in the first operation). However, a higher rate of very relevant postoperatively new permanent deficits was observed (aphasia: 3\%; paresis: 11\%; see Figure 2). Nonetheless, pre and postoperative KPS was also comparable in patients who underwent the first and repeated resection, which shows a persistent quality of life. In particular, our data on potential survival rates offers further evidence that reoperation of recurrent high-grade gliomas is beneficial. Although some authors stated that a second surgery for high-grade gliomas is comparable to conservative treatment [43], others provided evidence that surgery improves survival and quality of life in most patients [44].

Moreover, as mentioned in Table 3, only 2 patients with recurrent gliomas underwent both chemo- and radiotherapy as initial treatment. With regard to the supposed standardization of glioma therapy, this number is rather small and shows us that even more standardization or even centralized and not only interdisciplinary neurooncological tumor conferences might be indicated.

\section{Conclusions}

Our results showed that gliomas judged as non-resectable are potentially eligible for surgical resection. By using a multimodal approach including preoperative functional mapping, IOM, and awake craniotomy in some cases, achieving a high extent of resection at an acceptable rate of postoperative neurological deterioration is possible. Particularly after primary resection, no patient in our series suffered from any new permanent deficit. With regard to this data, patients with primarily rated "inoperable" gliomas should be referred to a specialized center to achieve the best oncological basis by surgical resection for an adjuvant therapy. Although the rate of new surgery-related neurological deficits is low and postoperative KPS and survival advocates for a surgical approach in the vast majority of cases, this decision 
must be discussed individually with every patient and in the context of a neuro-oncological conference including neurosurgical, neurologists, neuroradiologists, and radiotherapist. Moreover, neurosurgical centers with limited expertise on surgery of such highly eloquent lesions should strongly refer their patients for a second opinion to a specialized center.

\section{Abbreviations}

ANOVA: nonparametric one-way analysis of variance; BMRC: British Medical Research Council Scale; CMAP: compound muscle action potential; DCS: direct cortical stimulation; GBM: glioblastoma; GTR: gross total resection; IOM: intraoperative neuromonitoring; KPS: Karnofsky performance scale; MEP: motor evoked potentials; MRI: magnetic resonance imaging; nTMS: navigated transcranial magnetic stimulation; PET: positron emission tomography; SD: standard deviation; STR: subtotal resection.

\section{Competing interests}

The authors declare that they have no conflict of interest that affects this study. The study was completely financed by institutional grants from the Department of Neurosurgery. The authors report no conflict of interest concerning the materials or methods used in this study or the findings specified in this paper.

\section{Authors' contributions}

SK was responsible for data acquisition, handled the acquired data and performed literature research as well as statistical analyses. SK drafted the manuscript and its final revision. SK is also responsible for concept and design. LS was responsible for data acquisition, performed data analysis and clinical assessment. ES was responsible for data acquisition and approved and corrected the final version of the manuscript. DD was responsible for data acquisition, read and approved the final manuscript. TO and NB were responsible for data acquisition and approved and corrected the final version of the manuscript. JG and BM approved and corrected the final version of the manuscript. FR is responsible for the original idea, the concept, design, and statistical analyses. FR has also written and revised the manuscript, approved and corrected the final version. All authors read and approved the final manuscript.

\section{Authors' information}

All authors are strongly involved in the treatment of brain tumors including awake surgery, preoperative mapping, and intraoperative neuromonitoring in a specialized neurooncological center. BM is chairman and FR is vice chairman of the department.

\section{Author details}

'Department of Neurosurgery, Klinikum rechts der Isar, Technische Universität München, Ismaninger Str. 22, 81675 Munich, Germany. ${ }^{2}$ Department of Anesthesiology, Klinikum rechts der Isar, Technische Universität München, Ismaninger Str. 22, 81675 Munich, Germany.

Received: 10 July 2012 Accepted: 30 January 2013

Published: 2 February 2013

\section{References}

1. Yordanova YN, Moritz-Gasser S, Duffau H: Awake surgery for WHO Grade II gliomas within "noneloquent" areas in the left dominant hemisphere: toward a "supratotal" resection. Clinical article. J Neurosurg 2011, 115(2):232-239.

2. Stummer W, Pichlmeier U, Meinel T, Wiestler OD, Zanella F, Reulen HJ: Fluorescence-guided surgery with 5 -aminolevulinic acid for resection of malignant glioma: a randomised controlled multicentre phase III trial. Lancet Oncol 2006, 7(5):392-401.

3. Stummer W, Reulen HJ, Meinel T, Pichlmeier U, Schumacher W, Tonn JC, Rohde V, Oppel F, Turowski B, Woiciechowsky C, et al: Extent of resection and survival in glioblastoma multiforme: identification of and adjustment for bias. Neurosurgery 2008, 62(3):564-576.

4. Soffietti R, Baumert BG, Bello L, von Deimling A, Duffau H, Frenay M, Grisold W, Grant R, Graus F, Hoang-Xuan K, et al: Guidelines on management of low-grade gliomas: report of an EFNS-EANO Task Force. Eur J Neurol 2010, 17(9):1124-1133.

5. Laws ER Jr, Taylor WF, Clifton MB, Okazaki H: Neurosurgical management of low-grade astrocytoma of the cerebral hemispheres. JNeurosurg 1984, 61(4):665-673.

6. Schnell O, Scholler K, Ruge M, Siefert A, Tonn JC, Kreth FW: Surgical resection plus stereotactic 1251 brachytherapy in adult patients with eloquently located supratentorial WHO grade II glioma - feasibility and outcome of a combined local treatment concept. J Neurol 2008, 255(10):1495-1502.

7. Thon N, Eigenbrod S, Grasbon-Frodl EM, Lutz J, Kreth S, Popperl G, Belka C, Kretzschmar HA, Tonn JC, Kreth FW: Predominant influence of MGMT methylation in non-resectable glioblastoma after radiotherapy plus temozolomide. J Neurol Neurosurg Psychiatry 2011, 82(4):441-446.

8. Beauchesne P, Bernier V, Carnin C, Taillandier L, Djabri M, Martin L, Michel X, Maire JP, Khalil T, Kerr C, et al: Prolonged survival for patients with newly diagnosed, inoperable glioblastoma with 3-times daily ultrafractionated radiation therapy. Neuro Oncol 2010, 12(6):595-602.

9. Barrie M, Couprie C, Dufour H, Figarella-Branger D, Muracciole X, HoangXuan K, Braguer D, Martin PM, Peragut JC, Grisoli F, et al: Temozolomide in combination with BCNU before and after radiotherapy in patients with inoperable newly diagnosed glioblastoma multiforme. Ann Oncol 2005, 16(7):1177-1184.

10. Penfield W, Boldrey E: Somatic motor and sensory representation in the cerebral cortex of man as studied by electrical stimulation. Brain 1937, 60:389-443.

11. Krings T, Buchbinder BR, Butler WE, Chiappa KH, Jiang HJ, Cosgrove GR, Rosen BR: Functional magnetic resonance imaging and transcranial magnetic stimulation: complementary approaches in the evaluation of cortical motor function. Neurology 1997, 48(5):1406-1416.

12. Kombos T, Suess O, Funk T, Kern BC, Brock M: Intra-operative mapping of the motor cortex during surgery in and around the motor cortex. Acta Neurochir (Wien) 2000, 142(3):263-268.

13. Krieg SM, Buchmann NH, Gempt J, Shiban E, Meyer B, Ringel F: Diffusion tensor imaging fiber tracking using navigated brain stimulation-a feasibility study. Acta Neurochir (Wien) 2012, 154(3):555-563.

14. Krieg SM, Shiban E, Buchmann N, Gempt J, Foerschler A, Meyer B, Ringel F: Utility of presurgical navigated transcranial magnetic brain stimulation for the resection of tumors in eloquent motor areas. J Neurosurg 2012, 116(5):994-1001.

15. Krieg SM, Shiban E, Droese D, Gempt J, Buchmann N, Pape H, Ryang YM, Meyer B, Ringel F: Predictive value and safety of intraoperative neurophysiological monitoring with motor evoked potentials in glioma surgery. Neurosurgery 2012, 70(5):1060-1071.

16. Duffau H, Capelle L, Sichez J, Faillot T, Abdennour L, Law Koune JD, Dadoun S, Bitar A, Arthuis F, Van ER, et al: Intra-operative direct electrical stimulations of the central nervous system: the Salpetriere experience with 60 patients. Acta Neurochir (Wien) 1999, 141(11):1157-1167.

17. Duffau H, Lopes M, Arthuis F, Bitar A, Sichez JP, Van Effenterre R, Capelle L: Contribution of intraoperative electrical stimulations in surgery of low grade gliomas: a comparative study between two series without (1985-96) and with (1996-2003) functional mapping in the same institution. J Neurol Neurosurg Psychiatry 2005, 76(6):845-851.

18. De Benedictis A, Moritz-Gasser S, Duffau H: Awake mapping optimizes the extent of resection for low-grade gliomas in eloquent areas. Neurosurgeny 2010, 66(6):1074-1084. discussion 1084.

19. Haglund MM, Berger MS, Shamseldin M, Lettich E, Ojemann GA: Cortical localization of temporal lobe language sites in patients with gliomas. Neurosurgeny 1994, 34(4):567-576.

20. Keles GE, Lundin DA, Lamborn KR, Chang EF, Ojemann G, Berger MS Intraoperative subcortical stimulation mapping for hemispherical perirolandic gliomas located within or adjacent to the descending motor pathways: evaluation of morbidity and assessment of functional outcome in 294 patients. JNeurosurg 2004, 100(3):369-375.

21. Ojemann G, Ojemann J, Lettich E, Berger M: Cortical language localization in left, dominant hemisphere. An electrical stimulation mapping investigation in 117 patients. J Neurosurg 1989, 71(3):316-326.

22. Sanai N, Berger MS: Intraoperative stimulation techniques for functional pathway preservation and glioma resection. NeurosurgFocus 2010, 28(2):E1.

23. Sanai N, Polley MY, Berger MS: Insular glioma resection: assessment of patient morbidity, survival, and tumor progression. J Neurosurg 2010, 112(1):1-9. 
24. Huber WWD, Poeck K, Willmes K: Der Aachener Aphasie Test-Aufbau und Überprüfung der Konstruktion. Der Nervenarzt 1980, 51:475-482.

25. Neuloh G, Schramm J: Monitoring of motor evoked potentials compared with somatosensory evoked potentials and microvascular Doppler ultrasonography in cerebral aneurysm surgery. JNeurosurg 2004, 100(3):389-399.

26. Neuloh G, Pechstein U, Cedzich C, Schramm J: Motor evoked potential monitoring with supratentorial surgery. Neurosurgery 2007, 61(1 Suppl):337-346.

27. Scheufler KM, Zentner J: Total intravenous anesthesia for intraoperative monitoring of the motor pathways: an integral view combining clinical and experimental data. J Neurosurg 2002, 96(3):571-579.

28. Cedzich C, Taniguchi M, Schafer S, Schramm J: Somatosensory evoked potential phase reversal and direct motor cortex stimulation during surgery in and around the central region. Neurosurgery 1996, 38(5):962-970.

29. Taniguchi M, Cedzich C, Schramm J: Modification of cortical stimulation for motor evoked potentials under general anesthesia: technical description. Neurosurgery 1993, 32(2):219-226.

30. Sanai N, Chang S, Berger MS: Low-grade gliomas in adults. J Neurosurg 2011, 115(5):948-965

31. Sanai N, Mirzadeh Z, Berger MS: Functional outcome after language mapping for glioma resection. NEnglJMed 2008, 358(1):18-27.

32. Sanai N, Berger MS: Mapping the horizon: techniques to optimize tumor resection before and during surgery. ClinNeurosurg 2008, 55:14-19.

33. Martino J, Taillandier L, Moritz-Gasser S, Gatignol P, Duffau H: Re-operation is a safe and effective therapeutic strategy in recurrent WHO grade II gliomas within eloquent areas. Acta Neurochir (Wien) 2009, 151(5):427-436.

34. Chang EF, Clark A, Smith JS, Polley MY, Chang SM, Barbaro NM, Parsa AT, McDermott MW, Berger MS: Functional mapping-guided resection of lowgrade gliomas in eloquent areas of the brain: improvement of long-term survival. Clinical article. J Neurosurg 2011, 114(3):566-573.

35. Kombos T, Picht T, Derdilopoulos A, Suess O: Impact of intraoperative neurophysiological monitoring on surgery of high-grade gliomas. JClinNeurophysiol 2009, 26(6):422-425.

36. Neuloh G, Pechstein U, Schramm J: Motor tract monitoring during insular glioma surgery. JNeurosurg 2007, 106(4):582-592.

37. Chinot OL, Barrie M, Fuentes S, Eudes N, Lancelot S, Metellus P, Muracciole $X$, Braguer D, Ouafik L, Martin PM, et al: Correlation between O6-methylguanine-DNA methyltransferase and survival in inoperable newly diagnosed glioblastoma patients treated with neoadjuvant temozolomide. J Clin Oncol 2007, 25(12):1470-1475.

38. Picht T, Schulz J, Hanna M, Schmidt S, Suess O, Vajkoczy P: Assessment of the influence of navigated transcranial magnetic stimulation on surgical planning for tumors in or near the motor cortex. Neurosurgery 2012, 70(5):1248-1257.

39. Lioumis $\mathrm{P}$, Zhdanov A, Makela N, Lehtinen $\mathrm{H}$, Wilenius J, Neuvonen $\mathrm{T}$, Hannula H, Deletis V, Picht T, Makela JP: A novel approach for documenting naming errors induced by navigated transcranial magnetic stimulation. J Neurosci Methods 2012, 204(2):349-354.

40. Sollmann N, Picht T, Makela JP, Meyer B, Ringel F, Krieg SM: Navigated transcranial magnetic stimulation for preoperative language mapping in a patient with a left frontoopercular glioblastoma. J Neurosurg 2012, 118:175-179.

41. Dutzmann S, Gessler F, Bink A, Quick J, Franz K, Seifert V, Senft C: Risk of ischemia in glioma surgery: comparison of first and repeat procedures. J Neurooncol 2012, 107:599-607.

42. Takahashi S, Jussen D, Vajkoczy P, Picht T: Plastic relocation of motor cortex in a patient with LGG (low grade glioma) confirmed by NBS (navigated brain stimulation). Acta Neurochir (Wien) 2012, 154(11):2003-2008.

43. Clarke JL, Ennis MM, Yung WK, Chang SM, Wen PY, Cloughesy TF, Deangelis LM, Robins HI, Lieberman FS, Fine HA, et al: Is surgery at progression a prognostic marker for improved 6-month progression-free survival or overall survival for patients with recurrent glioblastoma? Neuro Oncol 2011, 13(10):1118-1124.

44. Xu JF, Fang J, Shen Y, Zhang JM, Liu WG, Shen H: Should we reoperate for recurrent high-grade astrocytoma? J Neurooncol 2011, 105(2):291-299.

doi:10.1186/1471-2407-13-51

Cite this article as: Krieg et al:: Surgery of highly eloquent gliomas primarily assessed as non-resectable: risks and benefits in a cohort study. BMC Cancer 2013 13:51.

\section{Submit your next manuscript to BioMed Central and take full advantage of:}

- Convenient online submission

- Thorough peer review

- No space constraints or color figure charges

- Immediate publication on acceptance

- Inclusion in PubMed, CAS, Scopus and Google Scholar

- Research which is freely available for redistribution 